
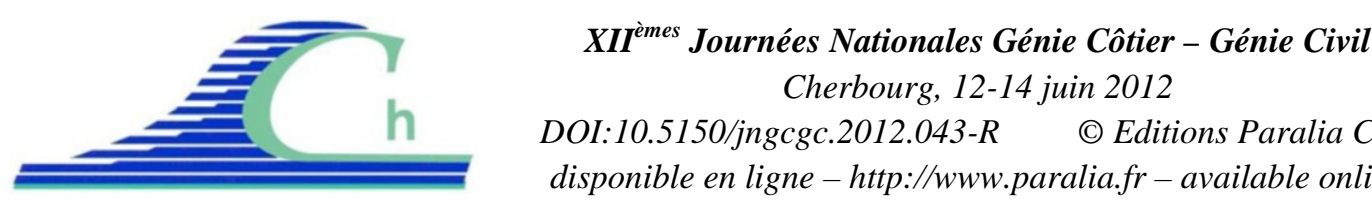

DOI:10.5150/jngcgc.2012.043-R @ Editions Paralia CFL disponible en ligne - http://www.paralia.fr - available online

\title{
Pour un socle commun des connaissances sur l'évolution du littoral : l'actualisation des "Catalogues sédimentologiques des côtes françaises"
}

\author{
Amélie ROCHE ${ }^{1}$, Catherine AZZAM ${ }^{2}$, Yann DENIAUD ${ }^{3}$, \\ Stéphanie DETOURBE ${ }^{4}$, Emmanuel DEVAUX $^{5}$, Carlos OLIVEROS $^{6}$, \\ Céline PERHERIN ${ }^{1}$, Frédéric RAOUT ${ }^{2}$, Jérôme REVEL ${ }^{7}$, \\ Céline TRMAL ${ }^{8}$, Pierre VIGNE ${ }^{4}$
}

1. Centre d'Études Techniques Maritimes Et Fluviales,

Technopôle Brest Iroise, 155, rue Pierre Bouguer, BP 5, 29280 Plouzané, France.

Amelie.Roche@developpement-durable.gouv.fr ;

Celine.Perherin@developpement-durable.gouv.fr

2. Ministère de l'Écologie, du Développement Durable, des Transports et du Logement

La Grande Arche, 92055 La Défense Cedex, France.

Direction Générale de la Prévention des Risques, Bureau des Risques Météorologiques.

Catherine.Azzam@developpement-durable.gouv.fr

Direction Générale de l'Aménagement, du Logement et de la Nature, Bureau du Littoral.

Frederic.Raout@developpement-durable.gouv.fr

3. Centre d'Études Techniques de l'Équipement Nord-Picardie,

151 rue de Paris, 02100 Saint Quentin, France.

Yann.Deniaud@developpement-durable.gouv.fr

4. Centre d'Études Techniques de l'Équipement Normandie-Centre,

10, Chemin de la Poudrière, 76121 Le Grand-Quevilly, France.

Stephanie.Detourbe@developpement-durable.gouv.fr ;

Pierre.Vigne@developpement-durable.gouv.fr

5. Centre d'Études Techniques de l'Équipement de l'Ouest,

Rue René Viviani, BP 46223, 44262 Nantes, France.

Emmanuel.Devaux@developpement-durable.gouv.fr

6. BRGM - Service Risques, Unité Risques Côtiers

3 avenue Claude Guillemin, BP 36009, 45060 Orléans Cedex 2, France.

c.oliveros@brgm.fr

7. Centre d'Études Techniques de l'Équipement du Sud-Ouest,

Rue Pierre Ramond, CS 60013, 33166 Saint-Médard-en-Jalles Cedex, France.

Jérôme.Revel@developpement-durable.gouv.fr

8. Centre d'Études Techniques de l'Équipement Méditerranée,

30 avenue Albert Einstein, CS 70499, 13791 Aix en Provence Cedex 03, France.

Céline.Trmal@developpement-durable.gouv.fr 


\section{Résumé :}

Le Grenelle de la Mer, le plan national d'adaptation au changement climatique et enfin la Stratégie nationale de gestion intégrée du trait de côte sont autant d'éléments du contexte actuel français rappelant la nécessité de disposer d'un socle commun de connaissances sur l'évolution du littoral. L'actualisation des "Catalogues sédimentologiques des côtes françaises", datant des années 1980, a ainsi été lancée en 2012 pour favoriser l'accès à tous à une donnée d'entrée nécessaire pour l'ensemble des politiques publiques liées à l'aménagement du littoral. Cette actualisation du fond et de la forme des catalogues comprendra notamment des bilans sur l'évolution pluri-annuelle passée des forçages hydro-sédimentaires, du trait de côte et de son artificialisation. Un questionnaire a été réalisé pour recueillir les besoins de la "communauté littorale" et s'assurer de l'opportunité de ce projet. Pour répondre à ces besoins, différentes actions sont lancées en parallèle du projet afin d'obtenir des données nationales valorisables dans ces catalogues, en particulier une étude sur l'évolution du trait de côte historique réalisée sur toutes les côtes françaises. Un zoom sur ce travail est ainsi proposé dans cet article, qui se veut être un appel à la mobilisation de cette communauté littorale pour valoriser ses travaux dans un socle de connaissances partagé et mis à disposition de tous.

\section{Mots-clés :}

Catalogues sédimentologiques - Facteurs hydrodynamiques - Bilans hydrosédimentaires - Evolution du littoral - Trait de côte - Artificialisation - Cartographie

\section{Introduction}

Depuis le lancement du Grenelle de la Mer en 2009, la nécessité de partager un socle de connaissances sur l'évolution du littoral français a été rappelée à maintes occasions. Dans ce contexte, le projet d'actualisation des "Catalogues sédimentologiques des côtes françaises", envisagé déjà depuis plusieurs années, est replacé au cœur d'actions stratégiques du Ministère de l'Écologie, du Développement Durable, des Transports et du Logement (MEDDTL). En effet, suite à la publication de "La gestion du trait de côte" (MEEDDM, 2010), le projet est clairement identifié en 2011 comme une action importante à mettre en œuvre dans le cadre de deux rapports majeurs : d'une part, du Plan national d'adaptation au changement climatique pour le littoral (ONERC, 2011) et, d'autre part, de la Stratégie nationale de gestion intégrée du trait de côte (MEDDTL, 2012). Dans son rapport remis à la Ministre de l'Écologie en 2011, le député de la Manche Alain COUSIN propose notamment pour cette stratégie nationale de "capitaliser les données existantes relatives aux facteurs d'évolution du trait de côte sous la forme de la mise à jour des catalogues sédimentologiques" pour "développer les outils de l'observation du trait de côte à l'échelle nationale et [...] pour prioriser l'action publique". Ce projet entre également dans le champ de la promotion des observatoires nationaux, tels les projets d'Observatoire national de la Mer et du Littoral, 


\section{XII ${ }^{\text {èmes }}$ Journées Nationales Génie Côtier - Génie Civil \\ Cherbourg, 12-14 juin 2012}

promu par le Grenelle de la Mer, d'Observatoire national des risques naturels et d'Observatoires de l'évolution du trait de côte. Cependant, ouvrages de référence il y a encore quelques années, les "Catalogues sédimentologiques" sont aujourd'hui peu connus et souvent devenus obsolètes par leurs données anciennes (ROCHE et al., 2011). Cet article présente donc les champs de connaissance couverts par les anciens catalogues et le projet d'actualisation, portant aussi bien sur la forme que sur le fond. L'organisation qui sera mise en place au cours de l'année 2012 pour sa réalisation est également abordée, ainsi qu'un zoom sur une action d'ores et déjà lancée: la digitalisation de traits de côte historiques pour cartographier l'évolution quantifiée du trait de côte sur l'ensemble des côtes françaises.

\section{Présentation des anciens catalogues}

Les "Catalogues sédimentologiques des côtes françaises" ont été réalisés au cours des années 1980 par le Service Technique Central des Ports Maritimes et des Voies Navigables (STCPMVN), ancêtre du CETMEF, pour le compte du Ministère chargé de la Mer. Ils comprenaient trois fascicules principaux relatifs à la Méditerranée, à la Mer du Nord et à la Manche est et à la Manche ouest et à l'Atlantique (resp. STCPMVN, 1984, 1986 et 1987a) et deux fascicules complémentaires spécifiques à la Corse (STCPMVN, 1987b) et à l'estuaire de la Gironde (STCPMVN, 1995). L'écriture des catalogues a été réalisée en partenariat avec les grands laboratoires d'hydraulique de l'époque, le Laboratoire National d'Hydraulique, le Laboratoire Central d'Hydraulique de France et SOGREAH, à partir de la bibliographie existante.

Ces fascicules font état sur chaque secteur :

a) d'une présentation du contexte général, précisant les données géographiques, géologiques, géomorphologiques, hydrographiques, climatiques et hydrologiques,

b) d'une synthèse des facteurs hydrodynamiques, dont les facteurs météorologiques (vents) et océanographiques (marée, niveaux de la mer, houles, courants),

c) d'une présentation des données sédimentologiques, comprenant la nature et répartition des sédiments, leur origine, leurs mouvements, la détermination des agents de transport prédominants et le sens et l'intensité des transits par mode de transport,

d) d'une présentation de l'évolution générale du littoral et des fonds, avec des bilans d'évolution de la "ligne de côte" et de l'impact quantifié des interventions humaines (inventaire des ouvrages maritimes existants, volumes dragués, etc.),

e) et d'un bilan sédimentaire général, précisant les modes et sens des transports, le bilan des apports, notamment des rivières, et de l'évolution du littoral.

Ces catalogues ont été des ouvrages de référence pour de nombreux gestionnaires, techniciens et scientifiques pour appréhender les phénomènes sur un secteur particulier d'étude. Ils restent à ce jour le seul exemple d'ouvrage de référence faisant un bilan de l'ensemble des études menées sur le littoral. 


\section{Projet d'actualisation}

L'actualisation des catalogues concerne donc autant la forme que le fond des documents. Depuis 2011, ce projet est en phase d'étude de faisabilité, pilotée par le CETMEF pour le Ministère de l'Écologie et en particulier la Direction Générale de l'Aménagement, du Logement et de la Nature. Dans ce cadre, un questionnaire a été réalisé et mis en ligne d'avril à juin 2011, à partir des sites du CETMEF (http://www.cetmef.developpementdurable.gouv.fr) et du Géolittoral (http://www.geolittoral.equipement.gouv.fr/). Plus de 400 réponses ont été reçues, comprenant plus de 100 propositions de contribution (ROCHE et al., 2011). Au-delà des bilans sur les forçages météo-marins et océaniques absolument nécessaires, quelques actions proposées ont été particulièrement soutenues, notamment :

- la mise à disposition de traits de côte historiques et d'un bilan de leur évolution passée, - un inventaire géolocalisé des ouvrages côtiers,

- un inventaire des tempêtes les plus marquantes sur le littoral et de leurs impacts,

- un recueil de photographies aériennes géoréférencées,

- un atlas de géomorphologie des côtes,

- et un bilan sur l'occupation du sol et l'artificialisation du littoral.

Sur la forme, un ouvrage plus pédagogique et accessible sur Internet, comprenant des cartographies interactives, a été recommandé. Le comité de pilotage du projet, qui se réunira au cours du premier semestre 2012, actera du site d'hébergement qui pourrait être le Géolittoral, en lien avec d'autres sites de référence tels Sextant de l'IFREMER (http://www.ifremer.fr/sextant), l'Observatoire du Littoral (http://www.littoral.ifen.fr) ou encore la base de données BOSCO (Base d'Observations pour le Suivi des Côtes: http://www.bosco.tm.fr/). Dans le respect de la directive européenne INSPIRE, l'ensemble des informations géographiques créées ou récoltées sera mis à disposition du public en visualisation et en téléchargement sur Internet, ce qui n'exclut pas la production d'un ouvrage papier ou distribué sur support type DVD.

Cependant, contrairement aux années 1980, la production d'éléments de connaissance sur l'évolution du littoral est aujourd'hui partagée, éclatée entre différents services de l'État, des collectivités ou syndicats mixtes, ou au sein des établissements publics et des universités. Le principal défi du projet sera d'associer l'ensemble des partenaires producteurs de données et détenteurs de la connaissance afin de la mettre à la disposition de tous. Ainsi, plusieurs comités seront montés afin d'assurer le suivi du projet au niveau national avec un comité de pilotage, appuyé par un comité scientifique, et au niveau local avec des comités de rédaction déclinés par grande province sédimentaire. Un comité spécifique à l'outre-mer sera également monté pour traiter ces territoires à part entière. 


\section{XII ${ }^{\text {èmes }}$ Journées Nationales Génie Côtier - Génie Civil \\ Cherbourg, 12-14 juin 2012}

\section{Zoom sur l'évaluation de l'évolution historique du trait de côte}

L'évaluation de l'évolution historique du trait de côte reposera sur deux approches : une approche qualitative à partir d'anciens documents cartographiques numérisés (cartes de Cassini, cadastres, notamment napoléonien, campagnes de Beautemps-Beaupré, cartes de l'Etat Major, etc.) et une approche quantitative à partir du relevé d'indicateurs de position de trait de côte sur des photographies aériennes orthorectifiées. Afin d'alimenter les futurs catalogues, une action d'évaluation quantitative de l'évolution historique du trait de côte, à partir d'indicateurs, a été lancée en 2011. C'est cette seconde approche qui est développée dans le cadre de cet article.

\subsection{Indicateurs de position du trait de côte sur support orthophotographique}

Les "indicateurs de position du trait de côte" sont ici des indices géomorphologiques, hydrodynamiques ou botaniques. Ils permettent en effet de rendre compte de l'évolution du littoral, au sens du recul, de la stabilité ou de l'accrétion des côtes, en s'affranchissant de la définition théorique du "trait de côte" donnée par le Service Hydrographique et Océanographique de la Marine ("la laisse des plus hautes mers dans le cas d'une marée astronomique de coefficient 120 et dans des conditions météorologiques normales"). Dans le cadre de ce travail, ces indicateurs doivent être facilement repérables sur des photographies aériennes anciennes orthorectifiées et comparables sur un même secteur à différentes époques (sur le siècle passé). Ainsi, des indicateurs adaptés aux contextes géomorphologique et hydrographique ont été proposés (cf. tableau 1).

Tableau 1. Synthèse des indicateurs de position de trait de côte proposés (CB : côte basse; CF : côte à falaise).

\begin{tabular}{|c|c|c|}
\hline éomorphologie & Régime & Indicateur(s) pertinent(s) \\
\hline CB rocheuse & tous & $\begin{array}{l}\text { Limite de végétation pérenne: supérieure des } \\
\text { lichens noirs ou inférieure de végétation terrestre }\end{array}$ \\
\hline bleuse & microtidal & Limite du jet de rive instantané \\
\hline use & mac & $\begin{array}{l}\text { Limite de la végétation pérenne (limite Chiendent } \\
\text { des sables/Oyat, limite inférieure de la végétation } \\
\text { terrestre) ou sommet de micro-falaise dunaire }\end{array}$ \\
\hline $\begin{array}{l}\text { B meuble - Cordon } \\
\text { le galets }\end{array}$ & macr & $\begin{array}{l}\text { Berme du cordon ou limite inférieure de la } \\
\text { végétation pérenne (Chou marin ou lichens) }\end{array}$ \\
\hline $\begin{array}{l}\text { CB meuble - Lagune } \\
\text { et marais maritime }\end{array}$ & macrotidal & $\begin{array}{l}\text { Limite du haut schorre (soit la limite inférieure de } \\
\text { la végétation pérenne terrestre) }\end{array}$ \\
\hline$C F$ - pente verticale & tous & Sommet de falaise \\
\hline$C F$ - pente oblique & tous & $\begin{array}{l}\text { Limite supérieure des lichens noirs, rupture de } \\
\text { pente ou limite de végétation terrestre }\end{array}$ \\
\hline
\end{tabular}


Les contextes géomorphologiques sont répartis d'une part entre les côtes basses et à falaises (définies par leur verticalité et leur hauteur supérieure à $2 \mathrm{~m}$ ), et d'autre part entre leurs composants meubles ou rocheux. Les côtes basses meubles recouvrent ainsi les côtes sableuses, avec ou sans cordon dunaire, les côtes à galets, notamment les cordons de galets, et les lagunes et marais maritimes. Les fonds de baie et plages de poche seront considérés localement comme des côtes basses meubles. Par contre, les massifs dunaires en érosion d'une hauteur supérieure à 2 mètres seront considérés comme des côtes à falaise meuble. Les côtes à falaise rocheuse regroupent les falaises "dures", peu évolutives, constituées de roches magmatiques, métamorphiques ou sédimentaires indurées (grès, calcaires, ...), et des falaises plus évolutives constituées de roches sédimentaires de type marno-calcaires, argiles ou de sédiments meubles. Les zones d'embouchures, estuaires et deltas, ne sont pas considérées comme une catégorie géomorphologique à part entière (les indicateurs retenus relèvent des types de côtes présentés ci-dessus). Une ligne transversale fictive pourra cependant être tracée entre les deux rives à une distance donnée à l'intérieur de l'embouchure dépendant de la zone d'action de la mer, déterminée à dires d'experts à partir de diverses informations (limite transversale de la mer, remontée de la marée saline, limite des masses d'eau, ...). Enfin, ces contextes géomorphologiques s'entendent hors zones influencées par des ouvrages côtiers, pour lesquelles un indicateur spécifique de "côte artificialisée" sera déterminé en fonction du type d'ouvrage, de la configuration des sites et du régime micro- ou macro-tidal.

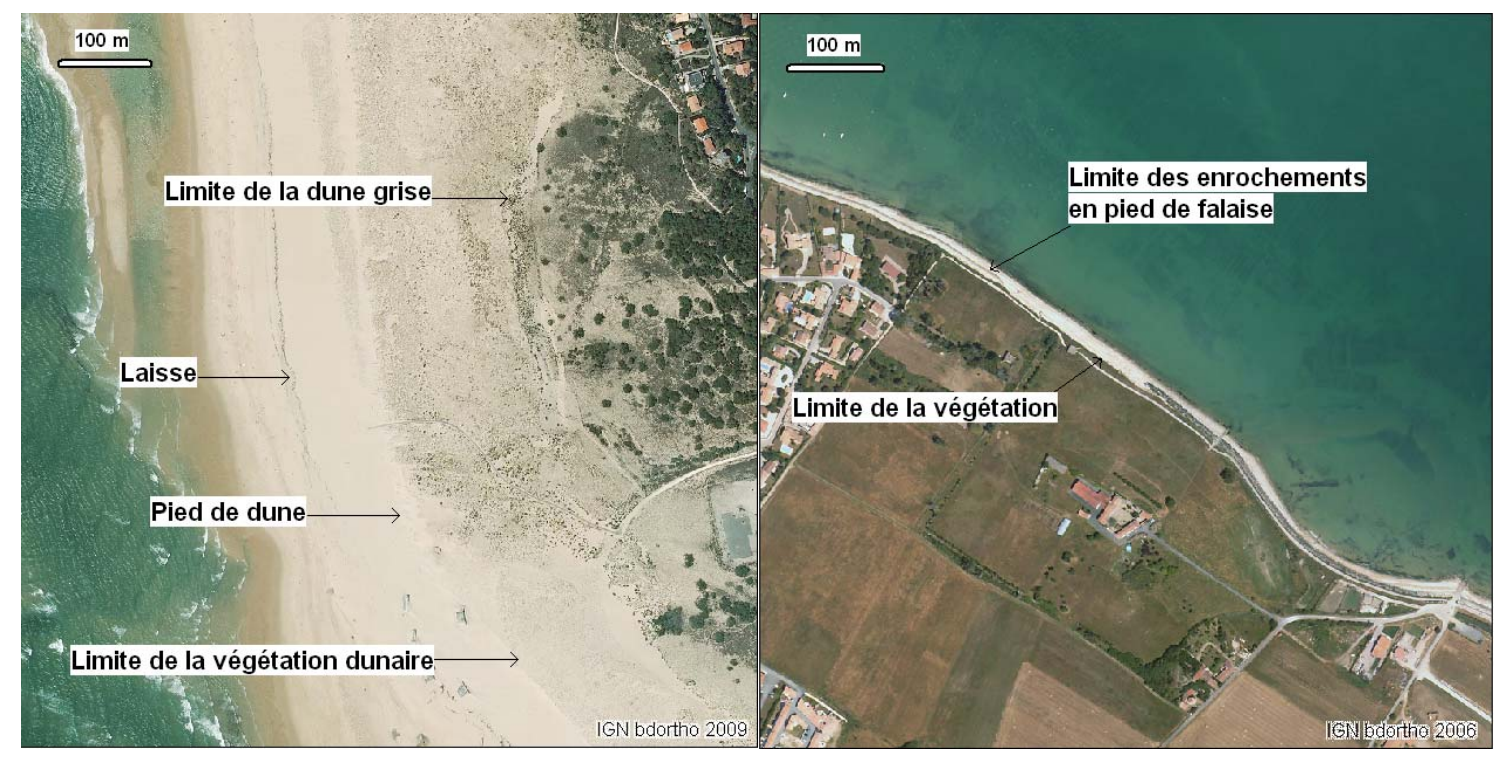

Figure 1. Exemples d'indicateurs géomorphologiques et botaniques visibles sur orthophotographie : (à gauche) indicateurs botaniques sur une côte basse avec massif dunaire au Cap Ferret (33) et (à droite) une côte à falaise sub-verticale sur l'Ile de Ré (fond de carte : BD Ortho® 2008 IGN). 


\section{XII ${ }^{\text {èmes }}$ Journées Nationales Génie Côtier - Génie Civil \\ Cherbourg, 12-14 juin 2012}

Les indicateurs doivent être nécessairement visibles sur orthophotographies, c'est pourquoi des indicateurs de type "botanique" ont généralement été préférés, ainsi que, pour les milieux en régime microtidal, des indicateurs de type "hydrodynamique", reflétant l'interface terre-mer. Les indicateurs botaniques correspondent à la "limite inférieure de végétation pérenne", souvent terrestre, qui n'est pas la même suivant les contextes géomorphologiques (cf. figure 1). Par exemple, les indicateurs botaniques suivants proviennent de BONNOT-COURTOIS \& LEVASSEUR (2002 en cours de réédition), et de l'ONF (1998):

- pour les côtes basses rocheuses (ou côtes à dénudation), il s'agira principalement de la limite du Lichen marin noir incrustant Verrucaria maura (si visible), sinon de la limite de la végétation terrestre ;

- pour les côtes basses meubles sableuses en régime macrotidal, il s'agira, lorsqu'elle existe, de la limite entre les espèces représentatives des milieux maritimes (par ex. le Chiendent des sables, Agropyron junceum) et continentaux (par ex. les prairies d'Oyat, Ammophila arenaria), ou de la limite inférieure de la végétation terrestre (NB : cette limite pourra correspondre le cas échéant à une micro-falaise d'érosion);

- pour les cordons de galets en régime macrotidal, il s'agira, lorsqu'elle existe, de la limite inférieure des lichens terrestres ou des plantes supérieures vivaces (en cas de stabilité du cordon, par exemple le Chou marin, Crambe maritima);

- enfin, pour les marais maritimes, si un ouvrage ne vient pas directement circonscrire le marais (cas des polders), il s'agira de plantes représentatives de la limite du hautschorre (par ex. prairies basses de Fétuque, Festuca rubra v. littoralis, pelouses à Bruyère de mer, Frankenia laevis, ou prairies à Chiendent piquant, Agropyron pungens).

Pour les côtes à falaises cependant, ces critères botaniques peuvent être difficiles à repérer sur des orthophotographies (couleurs pour les lichens, "verticalité" de la prise de vue, etc.). Pour les falaises verticales, le sommet de la falaise (y compris pour les falaises dunaires) sera relevé ; pour les autres falaises, ce seront soit les lichens marins noirs incrustants s'ils sont visibles, soit une rupture de pente marquée (par exemple dans le cas de "fausses falaises"), soit enfin la limite de la végétation terrestre.

Ainsi, sur chaque contexte géomorphologique, la position des indicateurs sera levée suivant une ligne continue ; à cette information linéaire sera associée une estimation de l'erreur par rapport à la position "réelle" du trait de côte en planimétrie. Sur certains secteurs fortement mobiles, la position linéaire du trait de côte sera peu pertinente pour rendre compte de l'évolution du littoral, notamment au niveau des flèches sableuses ou des zones de mangroves. Dans ces cas, un levé surfacique sera réalisé afin d'approcher l'évolution du rivage par un moyen plus représentatif de ces contextes.

Ce travail de digitalisation du trait de côte s'appuiera sur des campagnes de photographies aériennes orthorectifiées et géoréférencées disponibles. Seront exploitées en priorité les campagnes 2000 et 2011 de l'Orthophotographie littorale (en ligne sur le 
site Géolittoral) et la campagne "Ortho-historique" de photographies anciennes, hébergée à IFREMER, datant de 1919 à 1958. Ces campagnes ont été prises en condition de marée basse et ont une résolution respective de $50 \mathrm{~cm}$ pour l'Orthophotographie littorale et de 25 à $100 \mathrm{~cm}$ pour l'Ortho-historique. Ce levé sera réalisé par les Centres d'Etudes Techniques de l'Equipement à façade maritime à l'échelle du $1 / 2500^{\text {ème }}$ et en comparaison diachronique à partir de la campagne la plus précise et récente à disposition, c'est-à-dire l'Orthophotographie littorale 2011. Il aboutira à la production d'une polyligne de position du trait de côte pour chaque date, à laquelle seront associées des métadonnées utilisées par l'outil de calcul de l'évolution.

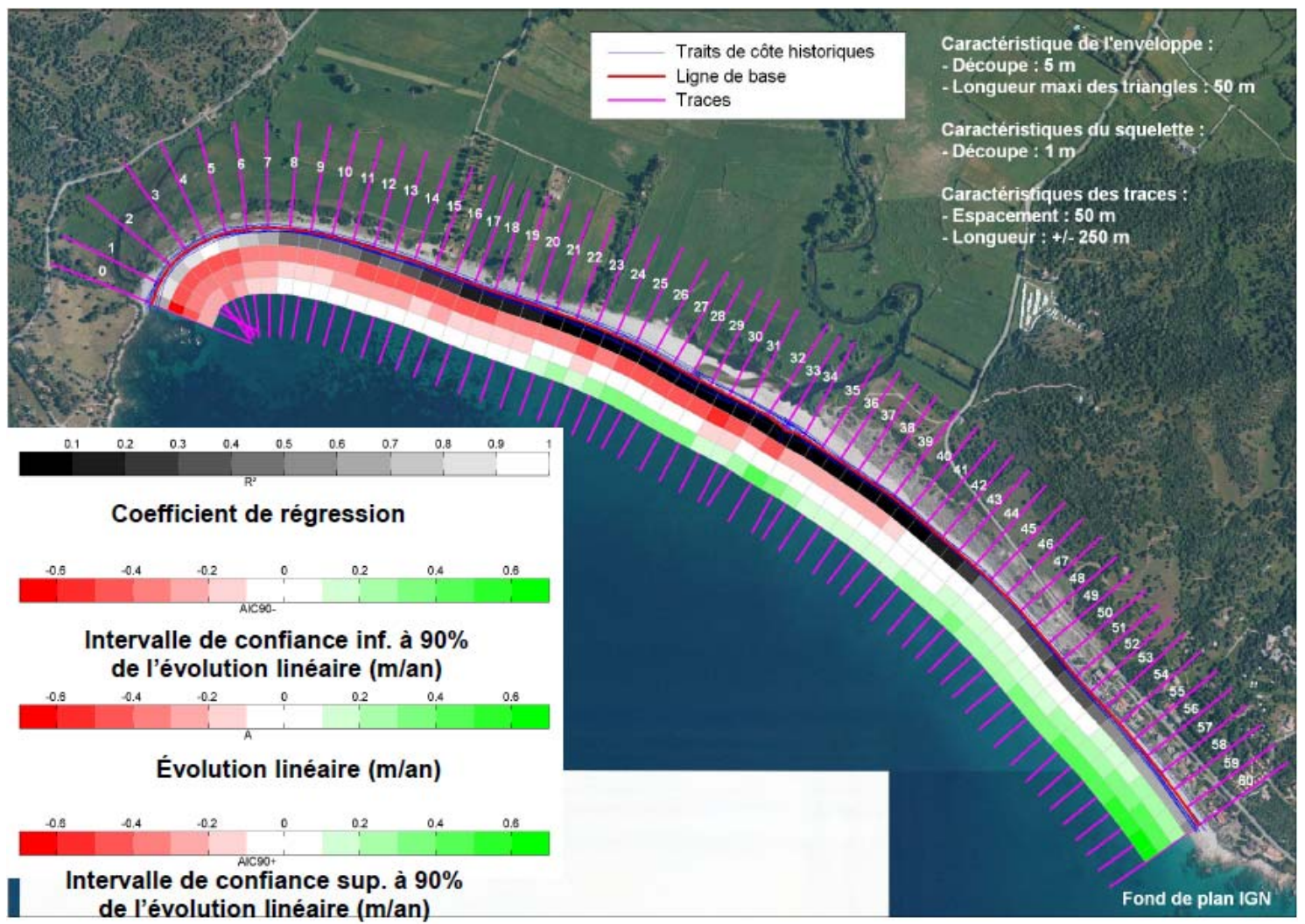

Figure 2. Exemple de restitution graphique de MobiTC et d'analyses possibles sur le site de Taravo (Corse-du-Sud). Fond de carte : BD Ortho ${ }^{\circledR} I G N$.

\subsection{Quantification automatique de leur évolution}

Les traits de côte ainsi obtenus seront traités avec un outil développé en parallèle dont l'objet est d'automatiser les calculs d'évolution du trait de côte, "MobiTC". A partir des polylignes créées et de leurs métadonnées, cet outil permet de générer un historique et des statistiques sur l'évolution du trait de côte à un pas d'espace modulable (http://www.wikhydro.org/index.php/MobiTC). Ces éléments sont visualisables avec un système d'information géographique. En particulier, les statistiques sur les positions passées (régressions linéaires et intervalles de confiance) permettent d'extrapoler les 


\section{XII ${ }^{\text {èmes }}$ Journées Nationales Génie Côtier - Génie Civil \\ Cherbourg, 12-14 juin 2012}

évolutions du trait de côte à des échéances futures (voir les exemples d'analyse en figure 2). Cet outil offre la possibilité de traiter automatiquement et rapidement de grandes longueurs de trait de côte ; actuellement le temps de traitement dépend plus du temps de validation et de caractérisation des données d'entrée que du temps de calcul, qui lui est fonction de la taille du domaine, de la discrétisation spatiale et du nombre de statistiques réalisées. Distribué gratuitement par le CETMEF sous couvert d'une licence d'utilisation, cet outil sera disponible en téléchargement sur Internet.

\section{Conclusions}

Les catalogues sédimentologiques des côtes françaises sont des ouvrages anciens qui ont longtemps été la référence des acteurs, gestionnaires et décideurs, du littoral. Le temps est venu de les actualiser afin d'offrir à nouveau un socle de connaissances partagé, couvrant à la fois les facteurs hydro-sédimentaires d'évolution que les facteurs anthropiques. A l'issue de l'étude de faisabilité en cours, le comité de pilotage du projet lancera officiellement les travaux en associant l'ensemble des acteurs locaux à la production de ces documents. Par ailleurs, des productions de données seront lancées au niveau national, à l'image de la réalisation de traits de côte historiques et la cartographie de l'évolution quantifiée du trait de côte sur l'ensemble des côtes françaises à l'aide de l'outil MobiTC. D'autres actions en cours ou à venir concernent l'établissement d'une bibliographie cartographiée des études de référence sur le littoral et la réalisation d'atlas de transits sédimentaires ou encore de bilans sur l'artificialisation du littoral.

\section{Remerciements}

L'équipe-projet de l'actualisation des catalogues sédimentologiques tient à remercier également Maëlle ALLAIN (DGALN), Virginie AMANT et Laurie-Anne HENO (CETE NC), François BOUTTES, Xavier KERGADALLAN, Bertrand MICHARD et Anne-Laure TIBERI-WADIER (CETMEF), Sébastien COLAS (CGDD), Guy DESIRE (CETE Ouest), Agathe LEBAILlEUX et Frédéric PONS (CETE Méd.), Cyril MALLET (BRGM/OCA) et Caroline MAURIN (CETE NP) pour leur contribution.

\section{Références bibliographiques}

BONNOT-COURTOIS C. (UMR PRODIG., EPHE), LEVASSEUR J.-E. (Université de Rennes 1) (2002, en cours de réédition). Reconnaissance de la limite terrestre du domaine maritime: intérêt et potentialités de critères morpho-sédimentaires et botaniques. CETMEF et RIVAGES, $149 \mathrm{p}$.

COUSIN A. (2011). Propositions pour une stratégie nationale de gestion du trait de côte, du recul stratégique et de la défense contre la mer, partagée entre l'État et les collectivités territoriales. MEDDTL, 61 p. [URL: http://www.developpementdurable.gouv.fr/IMG/pdf/rapport_final_SNGTC.pdf ].

MEEDDM -Ministère de l'Ecologie, de l'Energie, du Développement Durable et de la 
Mer- (2010). La gestion du trait de côte. Ed. Quae, 290 p. [URL : http://www.developpement-durable.gouv.fr/spip.php?page=Gestion_trait_cote ]

MEDDTL -Ministère de l'Ecologie, du Développement Durable, des Transports et du Logement- (2012). Stratégie nationale de gestion intégrée du trait de côte : vers la relocalisation des activités et des biens. MEDDTL, 20 p. [URL : http://www.developpement-durable.gouv.fr/Strategie-nationale-de-gestion.html ]

ONERC -Observatoire National des Effets du Réchauffement Climatique- (2011). Plan national d'adaptation au changement climatique. MEDDTL, 188 p. [URL : http://www.developpement-durable.gouv.fr/IMG/pdf/ONERC-PNACC-complet.pdf ]

ONF -Office National des Forêts- (1998). Guide de la flore des dunes littorales. De la Bretagne au sud des Landes. Ed. Sud Ouest, 168 p.

ROCHE A. (CETMEF), TRMAL C., LEBAILLEUX A. (CETE Méditerranée) (2011). Analyse des résultats de l'enquête sur l'actualisation des catalogues sédimentologiques des côtes françaises. CETMEF, 54 p. [URL : http://www.cetmef.developpementdurable.gouv.fr/analyse-des-resultats-de-l-enquete-a615.html ]

STCPMVN -Service Technique Central des Ports Maritimes et des Voies Navigables(1984). Catalogue sédimentologique des côtes françaises. Côtes de la Méditerranée, de la frontière espagnole à la frontière italienne. Secrétariat d'État auprès du Ministre des Transports, chargé de la Mer. Ed. Eyrolles, 300 p.

STCPMVN -Service Technique Central des Ports Maritimes et des Voies Navigables(1986). Catalogue sédimentologique des côtes françaises. Côtes de la Mer du Nord et de la Manche, de la frontière belge au Mont-Saint-Michel. Secrétariat d'État auprès du Ministre de l'Urbanisme, du Logement et des Transports, chargé de la Mer. Ed. Eyrolles, $407 \mathrm{p}$.

STCPMVN -Service Technique Central des Ports Maritimes et des Voies Navigables(1987a). Catalogue sédimentologique des côtes françaises. Côtes de la Manche et de l'Atlantique, de la baie du Mont-Saint-Michel à la frontière espagnole. Secrétariat d'État à la Mer. Ed. Eyrolles, 561 p.

STCPMVN -Service Technique Central des Ports Maritimes et des Voies Navigables(1987b). Catalogue sédimentologique des côtes françaises. Tome 10: la Corse. Ministère des Transports, $106 \mathrm{p}$.

STCPMVN -Service Technique Central des Ports Maritimes et des Voies Navigables(1995). Catalogue sédimentologique des côtes françaises. Côtes de la Manche et de l'Atlantique. Annexe I Littoral du département de la Gironde - Mise à jour 1983-1994. Secrétariat d'État aux Transports auprès du Ministre de l'Équipement, du Logement, des Transports et du Tourisme, $122 \mathrm{p}$.

TRMAL C., PONS F., SABATIER F. (2012). MobiTC, outil de calcul automatique de l'évolution historique du trait de côte : exemples sur 3 types de morphologies côtières. XII $^{\text {èmes }}$ Journées Nationales Génie Côtier - Génie Civil, Cherbourg, 9 p. doi:10.5150/jngcgc.2012.055-T 